\title{
Is routine urine dip stick testing justified in asymptomatic women in early pregnancy?
}

\author{
Jonathan Gaughran ${ }^{1 *}$, Bethany Chung², Tom Lyne², \\ Nuhaat Syeda $^{2}$, Daniel Field ${ }^{2}$, Judith Hamilton ${ }^{1}$
}

\begin{abstract}
${ }^{1}$ Department of Gynecology, Guys and St Thomas' NHS Trust, Westminster Bridge Road, London, SE1 7EH, UK
${ }^{2}$ Medical School, Kings College London, Strand, London, WC2R 2LS, UK
\end{abstract}

Received: 28 October 2020

Accepted: 03 December 2020

\section{*Correspondence:}

Dr. Jonathan Gaughran,

E-mail: Jonathan.gaughran@gstt.nhs.uk

Copyright: () the author(s), publisher and licensee Medip Academy. This is an open-access article distributed under the terms of the Creative Commons Attribution Non-Commercial License, which permits unrestricted non-commercial use, distribution, and reproduction in any medium, provided the original work is properly cited.

\begin{abstract}
Background: Routine urinalysis is commonly performed in early pregnancy units (EPUs) based on historic evidence that bacteriuria is linked to pyelonephritis, pre-term birth, mid trimester loss and low birth weight. Aim was to assess the cost and diagnostic yield of routine urinalysis in asymptomatic women in early pregnancy. A secondary outcome was the birth outcomes for women with proven bacteriuria.

Methods: Retrospective review of all urinalysis performed over 12 month period in a tertiary EPU and analysis of pregnancy outcomes in the proven bacteriuria group.

Results: 10,490 urinalyses performed at a cost of $£ 40,385.50 .1162(11 \%)$ positive urine dips; $68(0.6 \%)$ nitrite positive. 179 microscopy, culture and sensitivity performed at a cost of $£ 2593.71$. Of the 179,65 were culture positive giving a proven bacteriuria rate of $0.6 \%$. The most common pathogen was E-Coli. There were no recorded episodes of pyelonephritis and no statistical significance in the pre-term birth, mid trimester loss or low birth weight rate in the culture positive versus culture negative group.

Conclusions: The cost associated with routine urinalysis is significant and the diagnostic yield is extremely low. We did not identify an association between bacteriuria and adverse pregnancy outcomes. As such, urinalysis should only be performed in symptomatic/ high risk patients presenting to the EPU.
\end{abstract}

Keywords: Bacteriuria, Early pregnancy, Low birth weight, Miscarriage, Premature, Urinalysis

\section{INTRODUCTION}

The value of screening for Asymptomatic Bacteriuria (ASB) in pregnancy is increasingly debated. ASB is defined as significant levels of bacteria (greater than 105 colony forming units $/ \mathrm{ml}$ ) in the urine with no symptoms of a urinary tract infection (UTI). ${ }^{1}$ There is consensus that there is no benefit in screening the general population for ASB. ${ }^{1}$ Evidence emerged in the 1960 s and 70 s that untreated ASB in the first and second trimester could result in the development of pyelonephritis, low birth weight and pre-term birth. ${ }^{2}$ The prevalence of ASB in ambulatory, premenopausal women ranges from $2 \%$ to $10 \%{ }^{3}$ Due to the anatomical and physiological changes in pregnancy, ASB appears to be more prevalent with a higher incidence of progression to a symptomatic UTI. ${ }^{4}$ Many countries offer screening during routine antenatal care. In the United Kingdom (UK), the National Institute of health and care excellence (NICE) and the European Association of Urology both advise screening., ${ }^{5,6}$ This is based on a systematic review where all except three of the studies in favour of screening were from the 1960s and 70 s. GRADE software scored the evidence used by the review as 'low or very low quality' 2 More recently, a study with improved design showed that pregnant women with ASB had an increased risk of pyelonephritis but the disease course was limited and neonatal outcomes were no different from those of controls. ${ }^{7}$ In 2019 , a systematic 
review concluded that the low quality evidence available provided little confidence that screening and antimicrobial use in ASB reduced the incidence of pyelonephritis, preterm birth or low birth weight. ${ }^{8}$

Many women in the UK attend early pregnancy units (EPUs) before routine antenatal care has commenced. In most EPUs it is standard practice for all women to have a urinalysis, regardless of symptoms. The indications for sending a mid-stream urine (MSU) for microscopy, culture and sensitivity (M, C and S) vary and there appears to be a lower threshold for doing so than in nonpregnant women. The aim of this paper was to analyse the number and cost of urinalyses and MSUs over a twelve-month period and to assess the diagnostic yield, in a high risk, tertiary referral EPU. A Secondary outcome measure is the rate of adverse birth outcomes in the proven bacteriuria group.

\section{METHODS}

All women attending our tertiary referral EPU provided a urine sample for pregnancy testing and urinanalysis, with electronic documentation of outcome. The departmental protocol was to send any sample positive for the detection of nitrites for $\mathrm{M}, \mathrm{C}$ and $\mathrm{S}$ regardless of symptoms. A retrospective review of these electronic results was performed for all urinalyses undertaken in asymptomatic women between 7th July 2017 and 13th June 2018. Asymptomatic was defined as denial of dysuria, urinary frequency and/or offensive smelling urine. Lower abdominal pain in isolation was not considered symptomatic. Women with a negative urine pregnancy test or a diagnosis of retained products of conception (on ultrasound scan and clinical review) were excluded. If patients presented more than once to the unit (for serial blood tests or follow up scans) only the initial urinalysis was analysed. The exception was that if a subsequent visit during the same pregnancy generated a positive MSU it was this visit that was assessed.

Clinical records were reviewed in women with proven bacteriuria to see if the ASB had been treated, if there was evidence of cystitis or admission with pyelonephritis, and the subsequent birth outcome. For patients with proven bacteriuria and adverse birth outcome, clinical data were examined for risk factors independent of bacteriuria for this poor outcome. A Fischer's exact test was performed using Graph Pad Prism 8 to assess the difference between adverse outcomes in those with proven bacteriuria and those without.

\section{Urinalysis}

All women were provided with a clean collection pot and asked to void a MSU directly, with minimal handling. A registered nurse or nursing assistant used Roche Combutest ${ }^{\circledR} 7$ reagent strips as per the manufacturer's instructions. A subjective assessment of the reagent strip colour change compared to the provided colour scale was made and results documented contemporaneously. Samples for MSU were placed in a boric acid container and transferred to microbiology the same day.

\section{$M, C$ and $S$}

All samples were run through a. A.Menarini diagnostics SediMAX® automated cytometer with abnormal samples plated directly for sensitivity. Our hospital's policy is to culture all samples in pregnancy. Culture was performed using Biomérieux's Chromid $\AA$ Elite ${ }^{\circledR}$ plates. Incubation was performed for a minimum of 18-24 hours in aerobic conditions at 34-36oC. E-Coli identification was based on magenta growth on the plates, while all other microbes were identified using a Bruker Maldo-Tof mass spectrometer. Sensitivity was identified and quantified using a Biomérieux VITEK®2.

\section{RESULTS}

\section{Urinalysis}

During the study period, urinalysis was performed on 10,490 consecutive, unselected samples from women in early pregnancy, who were asymptomatic for a UTI. Ages ranged from 16-53 with a median of 32 years. Estimated gestations ranged from 3 weeks +2 days to 16 weeks +6 days with a median of 6 weeks +2 days.

Table 1: Details the $1162 / 10,490(16 \%)$ samples which were positive for either leucocytes, nitrites or both.

\begin{tabular}{|lll|}
\hline Urinalysis & Frequency & $\%$ \\
\hline Leu 1+ & 226 & 19 \\
\hline Leu 2+ & 397 & 34 \\
\hline Leu 3+ & 464 & 40 \\
\hline Leu 4+ & 7 & $<1$ \\
\hline Nit + & 44 & 4 \\
\hline Leu 1+ Nit & 3 & $<1$ \\
\hline Leu 2+ Nit & 9 & $<1$ \\
\hline Leu 3+ Nit & 12 & 1 \\
\hline Leu 4+ Nit & 0 & 0 \\
\hline Total & 1162 & \\
\hline
\end{tabular}

\section{$M, C$ and $S$}

Of the 10,490 urinalyses performed, $68(0.61 \%)$ were nitrite positive and sent for $\mathrm{M}, \mathrm{C}$ and $\mathrm{S}$, as per the departmental protocol. An additional 111 nitrite negative samples were also sent for M, C and S. 7 of these were leucocyte 4+ and the remainder (104) were leucocyte 3+. Of the 179 MSUs which underwent M, C and S, 65 $(36 \%)$ were culture positive, giving a proven bacteriuria rate of $0.6 \%(65 / 10,490)$ in the study. 
Table 2: FPR and PPV in nitrite positive and leucocyte positive sample sent for $M, C$ and $S$ and sensitivity and specificity of nitrites.

\begin{tabular}{|lllllll|}
\hline Urinalysis & MSU sent & Culture positive & FPR & PPV & Sensitivity & Specificity \\
\hline Nitrite positive & 68 & 41 & $27 \%$ & $73 \%$ & $63 \%$ & $76 \%$ \\
\hline Leucocytes only & 111 & 24 & $78 \%$ & $22 \%$ & - & - \\
\hline Total & 179 & 65 & & & & \\
\hline
\end{tabular}

Table 3: Types of bacteria cultured and their prevalence.

\begin{tabular}{|lll|}
\hline Bacteria & Prevalence & $\%$ \\
\hline Escherichia coli & 49 & 75 \\
\hline Klebsiella & 6 & 9 \\
\hline Group B Streptococcus & 4 & 6 \\
\hline Staphylococcus saprophyticus & 3 & 5 \\
\hline Proteus miribalis & 2 & 3 \\
\hline Enterococcus faecalis & 1 & 2 \\
\hline Total & 65 & 100 \\
\hline
\end{tabular}

Table 2 shows the Positive Predictive Values (PPV) and False Positive Rates (FPR) for each of nitrite positivity and the presence of leucocytes alone in predicting culture proven bacteriuria as well as the sensitive and specify for nitrite positivity in predicting bacterial colonisation. The bacteria cultured and their prevalence are described in Table 3.

\section{Clinical details}

There was documented evidence of appropriate antibiotic prescription in 61 of the 65 patients with a culture positive MSU. None of the 65 patients were admitted to our hospital with pyelonephritis.

\section{Cost of urine testing}

NICE estimates that each urinalysis costs $£ 3.85$ to perform (Appendix 1), equating to an expenditure of $£ 40,386.50 .11$ during the study period. A single M,C and $\mathrm{S}$ analysis costs $£ 14.49$ in our hospital laboratory giving a total bill of $£ 2593.71$ for the 179 samples sent. If only nitrite positive MSUs were analysed the cost would have been $£ 985.32$.

\section{Adverse birth outcomes}

Of the 65 patients with positive urine culture results birth outcomes are known for 35. Of the 35, 10 (29\%) had adverse birth outcomes. There were six pre term births $(17 \%)$, all in women who had received appropriate antibiotic treatment for their UTI. Independent risk factors for pre term birth were found in five of these six cases. There were 2 mid trimester losses (MTL) (6\%); one patient had received appropriate antibiotics and had no risk factors, the other patient had no documentation of treatment, but had a previous mid trimester loss believed to be secondary to cervical weakness. There were two low birth weight babies $(6 \%)$; both mothers had received antibiotic treatment for their UTI and had independent risk factors for this outcome. These outcomes are shown in Table 4.

Table 4: Adverse outcomes in positive urine culture group.

\begin{tabular}{|llll|}
\hline Adverse outcome & Number & Treated & $\begin{array}{l}\text { Risk } \\
\text { factors }\end{array}$ \\
\hline Pre-term birth & 6 & 6 & 5 \\
\hline MTL & 2 & 1 & 1 \\
\hline $\begin{array}{l}\text { Low birth } \\
\text { weight }\end{array}$ & 2 & 2 & 2 \\
\hline Total & 10 & & \\
\hline
\end{tabular}

Out of the $114 \mathrm{M}, \mathrm{C}$ and S samples that did not colonise bacteria birth outcomes are known for 73 patients. There were $16(21 \%)$ adverse birth outcomes. There was no statistical significance in the pre-term birth rate $(\mathrm{p}=0.62)$, MTL rate $(p=0.62)$, or low birth rate $(p>0.99)$ between those with a positive $\mathrm{M}, \mathrm{C}$ and $\mathrm{S}$ and those with a negative $\mathrm{M}, \mathrm{C}$ and $\mathrm{S}$.

\section{DISCUSSION}

Routine urinalysis is common in UK EPUs with the associated inconvenience (to patients and staff) and costs. Women are usually already providing a urine specimen for pregnancy testing and there is a tendency to also perform a full urinalysis, as do the majority of Emergency Departments and General Practices (GP). In later pregnancy, urine is dipped at every antenatal visit to screen for pre- eclampsia. ${ }^{5}$ This study is important as it provides data on a large number of consecutive, unselected women presenting to an early pregnancy unit who undergo routine urinalysis in the absence of symptoms of urinary tract disease. Limitations of the study include an incomplete data set due to women not continuing their obstetric care at our hospital meaning that birth outcomes and potential subsequent UTIs/pyelonephritis cannot be completely assessed and also the fact that $\mathrm{M}, \mathrm{C}$ and $\mathrm{S}$ was not performed in women with a negative urine dip meaning the sensitivity and specificity of leucocytes alone cannot be calculated.

Performing urinalysis in women asymptomatic for urinary disease is a form of screening. Pre-eclampsia does not occur in the first trimester. The first criterion any screening test must fulfil is that the condition being 
screened for should be an important health problem and its natural history understood. ${ }^{12}$ Urinary tract infections are one of the most common complications of pregnancy. The prevalence of ASB in pregnancy is believed to be higher than in non-pregnant women. Estimates of the overall incidence of pyelonephritis in pregnancy range from $0.5 \%$ to $2 \% .{ }^{13}$ The rate of pyelonephritis in untreated pregnant women with ASB is $30-40 \%$ versus 3$4 \%$ in those treated. ${ }^{13}$ There was no documented evidence of progression to cystitis or pyelonephritis in the four untreated, culture positive patients in our study. This is a very small number and the patients may have received treatment via their GP or at another hospital.

Historic and some more recent studies have found an association between symptomatic UTI/pyelonephritis and preterm birth and low birth weight. ${ }^{14,15}$ Others have suggested that sample heterogeneity and sources of potential bias invalidate the association. ${ }^{8,16}$ The efficacy of screening to prevent poor obstetric outcome is currently not proven. While we demonstrated no statistical significance in pre-term delivery or low birth weight in the culture positive versus culture negative group, 61 out of these 65 patients did receive appropriate antibiotics in a timely manner.

The pre-term birth weight was significantly higher for both culture positive and culture negative groups compared to the national average (17\% versus $8 \%){ }^{17}$ This may be biased due to a small sample size, and/or a reflection of the fact that our hospital is a tertiary referral unit for women at high risk of prematurity.

For successful screening the disease should have a detectable latent phase and treatment at this point should be better than later intervention. ASB is the latent phase prior to symptomatic infection. The risk of cystitis and pyelonephritis increases with gestational age, in keeping with the relative outflow obstruction caused by the developing fetus along with other physiological changes. $97.5 \%$ of pyelonephritis is diagnosed in the second or third trimester and as such an additional screening point in the first trimester seems unjustified. ${ }^{18}$

If screening for ASB in early pregnancy is to be performed, a safe, reliable and acceptable test should be available. Urinalysis is non-invasive and considered acceptable by patients. Interpretation and subsequent communication and/or documentation of results is time consuming and has been shown to be open to error. ${ }^{19}$

The decision by our unit to only send samples for $\mathrm{M}, \mathrm{C}$ and $\mathrm{S}$ that are positive for nitrites was based on evidence that leucocytes in isolation have a poor diagnostic yield for positive bacterial culture. ${ }^{9}$ In our large sample, only $11 \%$ of urines contained leucocytes and $0.6 \%$ were nitrite positive. The study was retrospective and in some situations individual clinicians (perhaps unaware of the departmental protocol or evidence) requested a number of nitrite negative samples were sent for $\mathrm{M}, \mathrm{C}$ and $\mathrm{S}$. This allowed us to examine the likelihood of a sample detecting bacteria if leucocyte positive but nitrite negative. Our results are in keeping with previous research demonstrating the false positive rate in nitrite negative samples to be unacceptably high, and the positive predictive value low. ${ }^{9}$ If urinalysis is performed in women asymptomatic for urinary disease, choosing to only culture nitrite positive samples seems justified.

Urine microscopy and culture is also non-invasive, and considered the gold standard for ASB detection. The pathogens cultured in our cohort were similar to other studies. E coli has been repeatedly shown to account for approximately $80 \%$ of all UTIs in pregnancy with GBS, proteus and klebsiella also common pathogens. ${ }^{2}$ Staphylococcus saprophyticus is the second most common cause of UTI in young sexually active women and therefore its detection was unsurprising. ${ }^{20}$

For a successful screening programme, there must be acceptable treatment available. Once ASB is detected the general consensus is to treat. Many antibiotics cross the placenta; are either FDA category $\mathrm{B}$ or $\mathrm{C}$; and some potentially have a teratogenic effect. ${ }^{13}$ The Oracle II trial suggested a link between the use of certain antibiotics and impaired neurological function in the fetus. The potential for intrauterine antibiotic exposure and subsequent immune dysfunction is gaining increasing evidence. $^{21,22}$ However the benefit of antibiotics in preventing pyelonephritis and its morbidity to mother and fetus must be balances with potential risk of antibiotic use.

Lastly, one of the difficulties associated with patients presenting to an early pregnancy unit is the frequency of lower abdominal pain as a symptom. For the purpose of this study lower abdominal pain in the absence of urinary symptoms was deemed asymptomatic. This was supported by literature suggesting that in the absence of lower urinary tract symptoms, the pre-test probability of having a UTI based purely on lower abdominal pain has a likelihood ration of only 1.1 versus 24.6 if dysuria and frequency are reported. ${ }^{23}$ As such, if practitioners are to disband screening in early pregnancy units, it would seem prudent to only perform them in women presenting with symptoms of lower urinary tract infection, sepsis or with recognisable risk factors such as recurrent UTI or structural urinary tract abnormalities.

\section{CONCLUSION}

Screening for ASB in asymptomatic women presenting to the early pregnancy unit has significant cost and poor diagnostic yield and as such should not be performed. We believe there is a role for urinalysis in women with symptoms or in those at increased risk of UTI.

Funding: No funding sources Conflict of interest: None declared

Ethical approval: Not required 


\section{REFERENCES}

1. NICE. Guideline (NG 109): Urinary tract infection (lower): antimicrobial prescribing 2018. Available at https://www.nice.org.uk/guidance/ng109. Accessed on 23 June 2020.

2. Smaill FM, Vazquez JC. Antibiotics for asymptomatic bacteriuria in pregnancy. Cochrane Database Syst Rev. 2015;8:CD000490.

3. Glaser AP, Schaeffer AJ. Urinary tract infection and bacteriuria in pregnancy. Urol Clin North Am. 2015;42:547-60.

4. Patterson TF, Andriole VT. Detection, significance, and therapy of bacteriuria in pregnancy. Update in the managed health care era. Infect Dis Clin North Am. 1997;11:593-608.

5. NICE. Guideline (CG 62): Antenatal care for uncomplicated pregnancies 2019. Available at https://www.nice.org.uk/guidance/cg62. Accessed on16 June 2020.

6. Bonkat G, Bartoletti RR, Bruyere F, Cai T, Geerlings SE, Koves B, et al. Guideline on urological infection. Available at https:// uroweb. org/ wp-content/ uploads/EAU-Guidelines-on-Urological-infections2019.pdf. Accessed on 18 June 2020.

7. Kazemier BM, Koningstein FN, Schneeberger C, Ott A, Bossuyt PM, Miranda E, et al. Maternal and neonatal consequences of treated and untreated asymptomatic bacteriuria in pregnancy: a prospective cohort study with an embedded randomised controlled trial. Lancet Infect Dis. 2015;15(11):132433.

8. Wingert A, Pillay J, Sebastianski M, Gates M, Featherstone R, Shave K, et al. Asymptomatic bacteriuria in pregnancy: systematic reviews of screening and treatment effectiveness and patient preferences. BMJ Open. 2019;9:e021347.

9. Little P, Turner S, Rumsby K, Warner G, Moore, M, Lowes JA, et al. Developing clinical rules to predict urinary tract infection in primary care settings: sensitivity and specificity of near patient tests (dipsticks) and clinical scores. British J General Practice. 2006;56(529):606-12.

10. Brown MA, Holt JL, Mangos GJ, Murray N, Curtis J, Homer C. Microscopic hematuria in pregnancy: Relevance to pregnancy outcome. Am J Kidney Dis. 2005;45(4):667-73.

11. NICE. Guideline 45 Routine preoperative testing for elective surgery. Available at https:// www. nice.org.uk/guidance/ng45/resources. Accessed on 19 June 2020

12. Wilson JMG, Jungner G. Principles and practice of screening for disease. World Health Organisation;
1968. Available at http:// www. who. int/bulletin/volumes/86/4/07-050112BP.pdf.

Accessed on 20 June 2020.

13. Rowińska MJ, Małyszko J, Wieliczko M. Urinary tract infections in pregnancy: old and new unresolved diagnostic and therapeutic problems. Arch Med Sci. 2015;11(1):67-77.

14. Gravett MG, Rubens CE, Nunes TM. GAPPS review group global report on preterm birth and stillbirth (2 of 7): discovery science. BMC Pregnancy Childbirth. 2010;10(1):1-26.

15. Sheiner E, Mazor E, Levy A. Asymptomatic bacteriuria during pregnancy. J Matern Fetal Neonat Med. 2009;22:423-7.

16. Beck S, Wojdyla D, Say L, Betran AP, Merialdi M, Requejo HJ, et al. The worldwide incidence of preterm birth: a systematic review of maternal mortality and morbidity. Bull World Health Organ. 2010;88:31-8.

17. Tommy's. Premature birth statistics. Available at https://www.tommys.org/our-organisation/why-weexist/premature-birth-statistics. Accessed on 19 October 2020.

18. Saleh P, Noshad H, Mallah F, Ramouz A. Acute pyelonephritis in pregnancy and the outcomes in pregnant patients. Arch Clin Infect Dis. 2015;10(3):e28886.

19. Delft S, Goedhart A, Spigt M, Pinxteren B, Wit N, Hopstaken R. Prospective, observational study comparing automated and visual point-of-care urinalysis in general practice. BMJ Open. 2016;6:e 011230.

20. Widerstöm M, Wiström J, Ferry S, Karlsson C, Monsen T. Molecular epidemiology of Staphylococcus saprophyticus isloalted from women with uncomplicated community acquired urinary tract infection. J Clin Microbiol. 2007;45:1561-4.

21. Kenyon S, Pike K, Jones DR, Brocklehurst P, Marlow N, Salt A, et al. Childhood outcomes after prescription of antibiotics to pregnant women with spontaneous preterm labour: 7-year follow-up of the ORACLE II trial. Lancet. 2008;372:1319-27.

22. Russel AR, Murch SH. Could peripartum antibiotics have delayed health consequences for the infant? BJOG. 2006;113:758-65.

23. Chu C, Lowder JL. Diagnosis and treatment of urinary tract infections across age groups. Am J Obst Gynecol. 2019;1:40-51.

Cite this article as: Gaughran J, Chung B, Lyne T, Syeda N, Field D, Hamilton J. Is routine urine dip stick testing justified in asymptomatic women in early pregnancy? Int J Reprod Contracept Obstet Gynecol 2021;10:17-21. 